\title{
WEB CREDIBILITY: A LITERATURE BASED OVERVIEW
}

\author{
R. SUJATHA ${ }^{1} \&$ P. NAGESWARA RAO ${ }^{2}$ \\ ${ }^{1}$ Research Scholar, External Part-Time, Bharathiar University, Coimbatore, Tamil Nadu, India \& \\ Librarian, Agurchand Manmull Jain College, Meenambakkam, Chennai, Tamil Nadu, India \\ ${ }^{2}$ Senior Librarian, Society for Electronic Transactions and Security (SETS), MGR
}

Knowledge City, CIT Campus, Taramani, Chennai, Tamil Nadu, India

\begin{abstract}
Web sites provide enormous information to the users, especially the academic web sites. The credibility of information provided by the academic web sites becomes more important. Numerous measures are available for measuring the website. But, the contents of web sites are considered as web credibility. The webometrics and web credibility are synonymously used by the research. This study identified the difference between webometrics and web credibility. Further, the study provides different types of web credibility and its taxonomy. Various evaluation methods of web credibility have also been suggested. The study provides various evaluation methods. Thus, a brief overview on web credibility has been presented in this paper.

KEYWORDS: Web Credibility; Web Site Evaluations, Academic Website, Human Method of Evaluation \& System Based Evaluation Methods
\end{abstract}

Received: Jul 05, 2017; Accepted: Jul 28, 2017; Published: Aug 07, 2017; Paper Id.: IJLSRAUG20176

\section{INTRODUCTION}

Today, web sites will propagate the progress of the organization, especially in the academic environment. They are the ambassadors between the user and academic institutions. These websites must be credible. These web sites are to be measured about the credibility. This study outlines a number of characteristics and attributes of the web credibility, specifically to academic sites, in order to show the quality requirement tree and a descriptive framework to specify them. These elements are used in a quantitative evaluation and comparison. The web credibility discussed in this paper has been indicative in nature and the intention is to provide web credibility in a nutshell.

\section{WEB CREDIBILITY DEFINITION}

Credibility is a subjective and an objective component. It is the believability of a source or message. It is evaluating the sources simultaneously in multiple dimensions. "Trustworthiness" and "Expertise" were the two main components of credibility. It is primarily to differentiate between trusts in relation to dependability. It is mainly connected to the idea of believability. Web credibility is one, which provides a trustworthy and provides knowledgeable information to those, who access the web site. (B.J. Fog)

Further it can be inferred the following terms as

- Credibility - Believability 
- $\quad$ Credibility People - Believable people

- Credible information - Information can be relied upon; Accurate and Correct; Believable information

- Credibility of a system based product - discussing the perception of credibility

Trust is a closely related concept. It is a belief, reliability and dependability of a person or information. (Fog \& Tseng, 1999). The credibility is a perceived quality that has to be induced in an object or in a person or in given information. It is accepted that credibility perceptions result from evaluating multiple dimensions simultaneously. Although the literature varies on how many dimensions contribute to credibility evaluations, it has identified the basic two key components of credibility viz. "Trustworthiness" and "expertise".

While evaluating credibility, one can arrive overall credibility by taking an assessment of expertise and trustworthiness of the website.

- Trustworthiness is a key element of credibility. It is nothing but well intentioned, truthful, unbiased, etc. The other dimensions of credibility were perceived goodness or morality of the website of source of information.

- Expertise - another dimension of credibility. It includes well intentioned, truthful unbiased etc. It also captures the skill of the source and perceived knowledge.

\section{OBJECTIVES}

The objectives of the study are

- To identify the difference between webometircs and web credibility

- To identify the different types of web credibility

- To know the web credibility taxonomy

- To identify the evaluation methods of web credibility

\section{Webometrics Vs Web Credibility}

Webometrics and Web Credibility are used synonymously by the researchers. In reality, there exist differences. It is very difficult to demarcate these two concepts. However, an attempt has been made to distinguish between Webometrics and Web Credibility and the same is shown in Table 1.

Table 1: Difference between Webometrics and Web Credibility

\begin{tabular}{|l|l|}
\hline \multicolumn{1}{|c|}{ Webometrics } & \multicolumn{1}{c|}{ Web credibility } \\
\hline $\begin{array}{l}\text { Quantitative studies of the web have been named } \\
\text { Webometrics }\end{array}$ & $\begin{array}{l}\text { Qualitative studies of the web have been named } \\
\text { web credibility }\end{array}$ \\
\hline It is utility based and design based & It is use based and user based \\
\hline$\bullet \quad$ Web link structure analysis & $\bullet$ Usability \\
(e.g. Hyperlink, Self-link and External link) & $\bullet$ Content, \\
$\begin{array}{l}\text { (e.g. Web usage analysis } \\
\text { expectations, browsing behavior), and }\end{array}$ & $\bullet \quad$ Functionality (services) \\
$\begin{array}{l}\bullet \quad \text { Web technology analysis (including search engine } \\
\text { performance) }\end{array}$ & $\bullet$ Navigation \\
\hline Evaluation can be made based on & Evaluations are user centric. Sociometric and \\
\hline
\end{tabular}




\begin{tabular}{|l|l|}
\hline Bibliometric/scientometric method & psychometric analysis can be used \\
\hline It is based on designer of the web site & $\begin{array}{l}\text { It depends on website provider, design and } \\
\text { content. }\end{array}$ \\
\hline
\end{tabular}

\section{Web Credibility Type}

Fog and Tseng (1999) provide a theoretical framework for studying Web credibility perceptions by separating them into four categories.

- Presumed Credibility

- Reputed Credibility

- Surface Credibility

- $\quad$ Earned Credibility

Presumed credibility judgments originate from assumptions made by the perceiver. The reputed credibility assessments depend on source labels, and the users make surface credibility judgments based on the layout or design of the website. The last and most reliable form of credibility is experienced credibility. The experienced credibility judgments are through the direct experience the user makes with a Web site.

\section{Web Credibility Taxonomy}

The web credibility can be viewed three dimensionally. One dimension represents personal/social. The other two dimensions are communication style and site/information. These three dimensions are shown in $\mathrm{X}, \mathrm{Y}$ and $\mathrm{Z}$ axis in the figure 1.

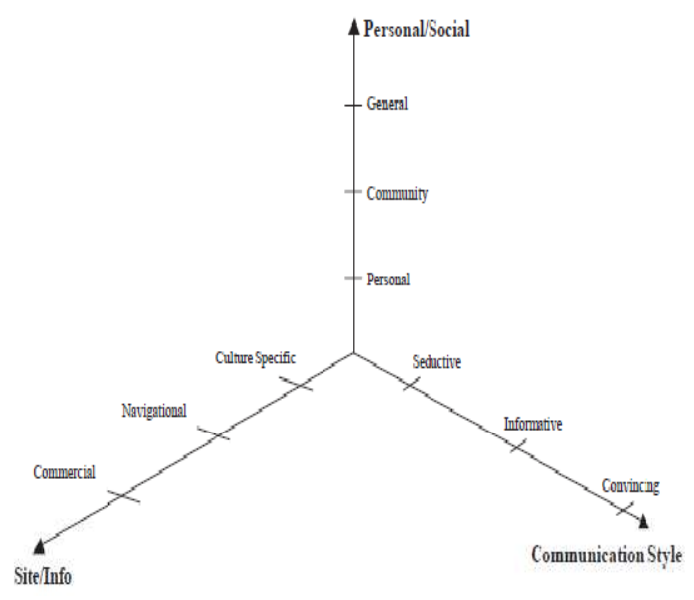

Figure 1: Taxonomy of Web Credibility

It can be seen from figure, Personal/Social dimension has three concepts namely general, community and personal. The communication style has three concepts such as selective, informative and convincing. The other dimension site/information has three concepts such as commercial, navigational and cultural specific.

\section{Web Credibility Grid}

The web credibility frame work can be combined with the four types of credibility such as presumed, reputed, surface and earned. Web credibility has many facets from brand perception of the organization to technical details 
including web site that will increasing the credibility. Therefore, their requires a strenuous effort in designing the web site. The web credibility grid in table 2 shows many facets, from brand perception of the technical details of the web site. The developers can use these grids to identify the cells they have control over on designing to boost credibility in the areas such as making the site easy to navigate. This grid also provides the entire organization to gain a better understanding of how its parts must work together to create and maintain a credible website.

Table 2: Web Credibility Grid

\begin{tabular}{|l|l|l|l|l|}
\hline & \multicolumn{1}{|c|}{ Presumed } & \multicolumn{1}{c|}{ Reputed } & \multicolumn{1}{c|}{ Surface } & \multicolumn{1}{c|}{ Experienced } \\
\hline Provider & $\begin{array}{l}\text { The provider is a } \\
\text { nonprofit } \\
\text { organization. }\end{array}$ & $\begin{array}{l}\text { The provider is } \\
\text { recognized as an } \\
\text { expert by others. }\end{array}$ & $\begin{array}{l}\text { Users are familiar } \\
\text { with the provider } \\
\text { outside of the Web }\end{array}$ & $\begin{array}{l}\text { Users with questions } \\
\text { receive quick and } \\
\text { helpful answers. }\end{array}$ \\
\hline $\begin{array}{l}\text { Content } \\
\text { - Information } \\
\text { - functionality }\end{array}$ & $\begin{array}{l}\text { The site has ads from } \\
\text { reputable companies. }\end{array}$ & $\begin{array}{l}\text { The content has been } \\
\text { approved by an } \\
\text { outside agency. }\end{array}$ & $\begin{array}{l}\text { The site appears to } \\
\text { have lots of relevant } \\
\text { information. }\end{array}$ & $\begin{array}{l}\text { The site's content has } \\
\text { always been accurate } \\
\text { and unbiased. }\end{array}$ \\
\hline $\begin{array}{l}\text { Design } \\
\text { - aesthetic } \\
\text { information } \\
\text { - technical }\end{array}$ & $\begin{array}{l}\text { The site has search } \\
\text { feature on the top of } \\
\text { the page. }\end{array}$ & $\begin{array}{l}\text { The site won an } \\
\text { award for technical } \\
\text { achievement. }\end{array}$ & $\begin{array}{l}\text { The site has a } \\
\text { pleasing visual } \\
\text { design. }\end{array}$ & $\begin{array}{l}\text { The site is easy to } \\
\text { navigate. }\end{array}$ \\
\hline
\end{tabular}

\section{Web Credibility Evaluation Method}

The web credibility study has been carried out based on the following methods:

- Check list method - (i) Nancy Everhart's Web Page Evaluation Worksheet and (ii) Benjamin Keevil's Usability Index Checklist for Websites.

- Guidelines Method - (i) Stanford Guidelines for Web Credibility and (ii) Nigel Bevan's usability guidelines for academic websites

- Theo Mandel's Golden Rule Method for user interface design

- Usability Heuristics - Jakob Nielsen

- 10 C's for Evaluating Internet Resources - Betsy Richmond

- Five W's of Website evaluation - Kathy Schrock

- The quality criteria for Website Excellence using a 100 factor scoring system.

Accessibility of web, either an expert or first time evaluator, primarily to identify the disability of people with

- Individual users Vs Broad range of users

- Fixing the known barriers before approaching the users

- Focus on potential areas of concern

\section{QUALITY FACTORS IN WEBSITE EVALUATION}

Quality factors in previous academic website evaluation research work, analyzing the characteristics of existing website quality models and website usability studies, showed that the most common website quality characteristics in the models are: 
- Usability

- Content

- Functionality (services)

- $\quad$ Efficiency and

- Navigation

Some of the quality characteristics in the models have similar semantics though they are given different names. For instance, the characteristic "services" in 2QCV3Q \& MiLE is similar to the functionality characteristic in the WebQEM model. Content in the Web-QEM and 7 Loci have a similar meaning with the Effective characteristic of the MINERVA model. A summary of the high level characteristics of the 4 website models reviewed are presented in Table 3 below:

Table 3: Previous Academic Websites' Evaluation Works

\begin{tabular}{|l|l|}
\hline \multicolumn{1}{|c|}{$\begin{array}{c}\text { Previous Academic Websites' } \\
\text { Evaluation Works }\end{array}$} & \multicolumn{1}{c|}{ Quality Factors } \\
\hline $\begin{array}{l}\text { L. Olsina, D. Godoy, G. Lafuente, and } \\
\text { G. Rossi }\end{array}$ & Usability, reliability, efficiency, Functionality \\
\hline S. Mustafa and L. Al-Zoua'bi, & $\begin{array}{l}\text { Content, organization, readability, navigation } \\
\text { and links, user interface design, performance } \\
\text { and effectiveness, educational information }\end{array}$ \\
\hline X. Wang and W. Huang & $\begin{array}{l}\text { Design, navigation, web technology, usability, } \\
\text { Functionality, content, branding }\end{array}$ \\
\hline $\begin{array}{l}\text { M. Lautenbach, I. Schegget, A. Schoute, } \\
\text { and C. Witteman }\end{array}$ & Survey ability, find ability \\
\hline C. Osborne and J. Rinalducci & Content, authority, organization, Accessibility \\
\hline H. M.Selim & $\begin{array}{l}\text { Consistency, flexibility and efficiency, } \\
\text { interactive facilities to help communications, } \\
\text { availability of essential course materials and } \\
\text { understandability }\end{array}$ \\
\hline $\begin{array}{l}\text { Mangala Hirwade (2006) } \\
\text { Jayasundari and R. Jeyshankar (2017) }\end{array}$ & $\begin{array}{l}\text { Contact details, Authority details, Currency } \\
\text { details, Website design factors, Content, } \\
\text { Coverage, Status, Activities Ease of Navigation, } \\
\text { Provision of User Support links, Expertise } \\
\text { criteria, Library related factors, Links to other } \\
\text { websites and Download speed }\end{array}$ \\
\hline
\end{tabular}

\section{Evaluation Using Human vs. Computers}

Normally, websites are evaluated using manual method and computer method. The various techniques used in evaluation are shown in Table 4.

Table 4: Evaluation Technique - Human Vs Computer

\begin{tabular}{|c|l|l|}
\hline S. No. & \multicolumn{1}{|c|}{ Evaluation Techniques Using Humans } & \multicolumn{1}{|c|}{ Evaluation Techniques Using Computers } \\
\hline 1 & Checklist Approach & Scaffolding tool Approach \\
\hline 2 & Prominence - Interpretation Factors & Credibility seal programs \\
\hline 3 & Contextual Approach & Digital signatures \\
\hline 4 & Cognitive Approach & Machine Learning \\
\hline 5 & Motivation - Centered Approach & Platform for Internet content selection \\
\hline 6 & Social and Heuristic Approach & Collaborative filtering and peer-review \\
\hline
\end{tabular}




\begin{tabular}{|l|l|l|}
\hline \multicolumn{2}{|c|}{ Table 4: Contd., } \\
\hline 7 & & Semantic Web \\
& & Visual cues approach \\
\cline { 3 - 3 } & & Credibility ratings systems \\
\hline
\end{tabular}

\section{EVALUATION CRITERIA}

The criteria for evaluation were made based on the high level quality factor, and their sub level quality factors are shown in Table 5

Table 5: Evaluation Criteria

\begin{tabular}{|c|l|l|}
\hline S. No. & $\begin{array}{c}\text { High Level } \\
\text { Quality Factor }\end{array}$ & \multicolumn{1}{|c|}{ Sub Level Quality Factors } \\
\hline 1 & Usability & $\begin{array}{l}\text { Understandability; Learn ability; } \\
\text { interactivity; Operability; } \\
\text { Interface attractiveness and } \\
\text { Multiple Language support }\end{array}$ \\
\hline 2 & Content & $\begin{array}{l}\text { Relevance of information; } \\
\text { Accuracy of information } \\
\text { Up-to-date of information; } \\
\text { Authority and Identity }\end{array}$ \\
\hline 3 & Reliability & $\begin{array}{l}\text { Fault tolerance; Recoverability and } \\
\text { Availability }\end{array}$ \\
\hline 4 & Efficiency & Time Behavior; Accessibility \\
\hline 5 & Functionality & Navigation; Search and Suitability \\
\hline
\end{tabular}

\section{Check List Methods}

There exist several check list methods. The check list methods normally used for evaluating the web site were:

- Stanford Guidelines for Web Credibility;( www.webcredibility.org/guidelines)

- 39 Factors: Website Credibility Checklist; http://conversionxl.com/website-credibility-checklist-factors/

- Cornell University Library Olin and Uris Libraries for Evaluating Web Sites: Criteria and Tools https://olinuris.library.cornell.edu/ref/research/webeval.htmland

- The Ten Nielsen's Heuristics for User Interface Design and Evaluation http://www.useit.com/papers/heuristic/heuristic_list.html

Based on the check list method stated above, a model check list has been developed, and the same has been indicated as Gopal Krishnan and Nageswara Rao’s Evaluating Academic Web Sites in Appendix -A

\section{CONCLUSIONS}

This study identified the difference between webometrics and web credibility. Further, the study provides different types of web credibility and its taxonomy. The study provides various evaluation methods. Based on various evaluation methods, web credibility evaluation check list for academic website has been suggested.

\section{REFERENCES}

1. Bouch, A. Kuchinsky, A., \& Bhatti, N. (2000). Quality is in the eye of the beholder: meeting user's requirements for internet 
quality of services. Paper presented at the ACM Conferences on Human Factors in Computing Systems (CHI), 297-304.

2. Ceaparu, I., Demner, D., Hung, E., Zhao, H., \& Shneiderman, B. (2002). In Web we trust: establishing strategic trust among online customers. In R.Rust \& P.Kannan (Eds.), E-Services. Armonk, NY: E-Sharpe Publishers, 90-107.

3. Diller, S., Lin, L., \& Tashijian, V. (2003). The evolving role of security, privacy, and trust in a digitized world. In J. Jacko \& A. Sears (Eds.). The Handbook of Human-Computer Interaction. Mahwah, NJ: Lawrence Erlbaum Associates, 1213-1225.

4. Fogg, B.J. and Tseng, H. 1999. The elements of computer credibility. Available at http://captology.stanford.edu/pdf/p80fogg.pdf. Accessed on Oct 04,2016

5. Fog, B.J., (2002). How do people evaluate a website's credibility? Available at http://www.consumerwebwatch.org/pdfs/stanfordPTL.pdf Accessed on Oct 04,2016

6. Fog, B.J., (2003). Prominence-Interpretation Theory: Explaining How People Assess Credibility Online. Retrieved on November 2,2016 from ACM Digital Library.

7. Hochheiser, H., \& Shneiderman, B. (2001). Universal usability statements: Marking the trail for all users. Interactions, 16-18.

8. http://www.kidnapcustomers.com/how-tomake-a-website/website-credibility/Retrieved on Oct 3, 2016.

9. Jacko, J., Sears, A., \& Borella, M. (2000). The effect of network delay and media on user perceptions of web resources. Behaviour and Information Technology, 19(6), 427-439.

10. Lazar, J. (2006). Web Usability: A User-Centered Design Approach. Boston: Addison-Wesley.

11. Lazar, J., \& Sears, A. (2005). Design of E-Business Web Sites. In G. Salvendy (Ed), Handbook of Human Factors and Ergonomics. Hoboken, NJ: John Wiley \& Sons.

12. Lohse, G.; Spiller, P., 1998, "Electronic Shopping", CACM 41,7 (Jul-98); pp. 81-86.

13. Luo, W., \& Najdawi, M. (2004). Trust building measures: A review of consumer health portals. Communications of the ACM, 47(1), 109-113.

14. Mangala, Anil Hirwade (2006). Websites of Indian Universities: An evalua tion. 2006. Bombay: Himalaya Publishing House.

15. McCall, J.A; Richards, P.K.; Walters, G.F.; 1977, “Factors in Software Quality“, RADC TR-77-369.

16. Metzger, M., Flanagin, A., Eyal, K., Lemus, D., \& McCann, R. (2003). Credibility for the $21^{\text {st }}$ Century: Integrating Perspectives on source, message, and media credibility in the contemporary media environment. In P. Kalbfleisch (Ed.), Communication Yearbook 27, Mahwah, NJ: Lawrence Erlbaum Associates, 293-335.

17. Miller, J.R.; 1970, “Professional Decision-Making”, Praeger Publisher.

18. Milne, G., \& Culnan, M. (2002). Using the Content of Online Privacy Notices to Inform Public Policy: A Longitudinal Analysis of the 1998-2001 U.S. web Surveys. The Information society, 18(5), 345-360.

19. Nielsen, Jakob; The Alertbox, http://www.useit.com/alertbox/

20. Olsina, L, 1998, "Building a Web-based Information System applying the Hypermedia Flexible Process Modeling Strategy"; 1 st International Workshop on Hypermedia Development, at ACM Hypertext 98, Pittsburgh, US (The paper is available at http://ise.ee.uts.edu.au/hypdev/).

21. Olsina, L., 1998, "Web-site Quantitative Evaluation and Comparison: a Case Study on Museums", ICSE '99 Workshop on Software Engineering over the Internet

22. Olsina, L., Rossi, G.; 1998, "Toward Web-site Quantitative Evaluation: defining Quality Characteristics and measurable 
Attributes", Submitted paper to WebNet '99 Conference.

23. Ramsay, J., Barbesi, A., and Preece, J. (1998). A Psychological investigation of long retrieval times on the World Wide Web. Interacting with Computers, 10(1), 77-86.

24. Zhang, P. and Dran, G. (2000). Satisfiers and dissatisfies: A two-factor model for Website design and evaluation. Journal of the American Society for Information Science, 51 (14), 1253-1268.

\section{APPENDIX -A}

Gopal Krishnan and Nageswara Rao's Evaluating Academic Web Sites: A Checklist

Table 6: General Features of the Website

\begin{tabular}{|l|l|l|l|l|l|}
\hline Use of Websites & $\begin{array}{l}\text { Difficult } \\
\square\end{array}$ & $\begin{array}{l}\text { Very } \\
\text { Difficult } \\
\square\end{array}$ & $\begin{array}{l}\text { Not so difficult } \\
\square\end{array}$ & $\begin{array}{l}\text { Simple } \\
\square\end{array}$ & $\begin{array}{l}\text { Easy } \\
\square\end{array}$ \\
\hline About its progress & $\begin{array}{l}\text { Unhelpful } \\
\square\end{array}$ & $\begin{array}{l}\text { Less } \\
\text { cooperative } \\
\square\end{array}$ & $\begin{array}{l}\text { To some extent } \\
\text { cooperative } \\
\square\end{array}$ & $\begin{array}{l}\text { Co-operative } \\
\square\end{array}$ & $\begin{array}{l}\text { Helpful } \\
\square\end{array}$ \\
\hline $\begin{array}{l}\text { Learning to use the } \\
\text { websites }\end{array}$ & $\begin{array}{l}\text { Very } \\
\text { Difficult } \\
\square\end{array}$ & $\begin{array}{l}\text { Difficult } \\
\square\end{array}$ & $\begin{array}{l}\text { Not so difficult } \\
\square\end{array}$ & $\begin{array}{l}\text { Simple } \\
\square\end{array}$ & $\begin{array}{l}\text { Easy } \\
\square\end{array}$ \\
\hline $\begin{array}{l}\text { Learning to use the } \\
\text { websites }\end{array}$ & $\begin{array}{l}\text { Very } \\
\text { Difficult } \\
\square\end{array}$ & $\begin{array}{l}\text { Difficult } \\
\square\end{array}$ & $\begin{array}{l}\text { Not so difficult } \\
\square\end{array}$ & $\begin{array}{l}\text { Simple } \\
\square\end{array}$ & $\begin{array}{l}\text { Easy } \\
\square\end{array}$ \\
\hline $\begin{array}{l}\text { Exploring new } \\
\text { features by trial and } \\
\text { error }\end{array}$ & $\begin{array}{l}\text { Very } \\
\text { Difficult } \\
\square\end{array}$ & $\begin{array}{l}\text { Difficult } \\
\square\end{array}$ & $\begin{array}{l}\text { Not so difficult } \\
\square\end{array}$ & $\begin{array}{l}\text { Simple } \\
\square\end{array}$ & $\begin{array}{l}\text { Easy } \\
\square\end{array}$ \\
\hline $\begin{array}{l}\text { Performing tasks is } \\
\text { straightforward }\end{array}$ & $\begin{array}{l}\text { Never } \\
\square\end{array}$ & $\begin{array}{l}\text { Sometimes } \\
\square\end{array}$ & $\begin{array}{l}\text { Forever } \\
\square\end{array}$ & $\begin{array}{l}\text { At all times } \\
\square\end{array}$ & $\begin{array}{l}\text { Always } \\
\square\end{array}$ \\
\hline Website Loading & $\begin{array}{l}\text { Very Slow } \\
\square\end{array}$ & $\begin{array}{l}\text { Slow } \\
\square\end{array}$ & $\begin{array}{l}\text { Quick } \\
\square\end{array}$ & $\begin{array}{l}\text { Fast Enough } \\
\square\end{array}$ & $\begin{array}{l}\text { High Speed } \\
\square\end{array}$ \\
\hline $\begin{array}{l}\text { Designed for all levels } \\
\text { of users }\end{array}$ & $\begin{array}{l}\text { Never } \\
\square\end{array}$ & $\begin{array}{l}\text { Sometimes } \\
\square\end{array}$ & $\begin{array}{l}\text { Forever } \\
\square\end{array}$ & $\begin{array}{l}\text { At all times } \\
\square\end{array}$ & $\begin{array}{l}\text { Always } \\
\square\end{array}$ \\
\hline
\end{tabular}

Table 7: Common Features of the Website

\begin{tabular}{|l|l|l|l|l|l|}
\hline Opinion of Websites & Frustrating & $\begin{array}{l}\text { Very Irritating } \\
\square\end{array}$ & $\begin{array}{l}\text { Not so Irritating } \\
\square\end{array}$ & $\begin{array}{l}\text { Fulfilling } \\
\square\end{array}$ & $\begin{array}{l}\text { Satisfying } \\
\square\end{array}$ \\
\hline $\begin{array}{l}\text { Use of terms } \\
\text { throughout websites }\end{array}$ & $\begin{array}{l}\text { Inconsistent } \\
\square\end{array}$ & $\begin{array}{l}\text { Inherent } \\
\square\end{array}$ & $\begin{array}{l}\text { Uniform } \\
\square\end{array}$ & $\begin{array}{l}\text { Coherent } \\
\square\end{array}$ & $\begin{array}{l}\text { Consistent } \\
\square\end{array}$ \\
\hline Prompts for input & $\begin{array}{l}\text { Confusing } \\
\square\end{array}$ & $\begin{array}{l}\text { Unclear } \\
\text { Error Messages }\end{array}$ & $\begin{array}{l}\text { Not so clear } \\
\square\end{array}$ & $\begin{array}{l}\text { Clear } \\
\square\end{array}$ & $\begin{array}{l}\text { Very Clear } \\
\square\end{array}$ \\
\hline $\begin{array}{l}\text { Help messages on } \\
\text { the Screen }\end{array}$ & $\begin{array}{l}\text { Less } \\
\text { cooperative } \\
\square\end{array}$ & $\begin{array}{l}\text { To some extent } \\
\text { cooperative } \\
\square\end{array}$ & $\begin{array}{l}\text { Co- } \\
\text { operative } \\
\square\end{array}$ & $\begin{array}{l}\text { Helpful } \\
\square\end{array}$ \\
\hline Compatibility & $\begin{array}{l}\text { Less } \\
\text { cooperative } \\
\square\end{array}$ & $\begin{array}{l}\text { To some extent } \\
\text { cooperative } \\
\square\end{array}$ & $\begin{array}{l}\text { Co- } \\
\text { operative } \\
\square\end{array}$ & $\begin{array}{l}\text { Helpful } \\
\square\end{array}$ \\
\hline
\end{tabular}

Table 8: Website Design

\begin{tabular}{|l|l|l|l|l|l|}
\hline Views of websites & $\begin{array}{l}\text { Terrible } \\
\square\end{array}$ & $\begin{array}{l}\text { Horrible } \\
\square\end{array}$ & $\begin{array}{l}\text { To some extent } \\
\text { horrible } \\
\square\end{array}$ & $\begin{array}{l}\text { Pleasing } \\
\square\end{array}$ & $\begin{array}{l}\text { Wonderful } \\
\square\end{array}$ \\
\hline Images in website & $\begin{array}{l}\text { Dull } \\
\square\end{array}$ & $\begin{array}{l}\text { Boring } \\
\square\end{array}$ & $\begin{array}{l}\text { Not so boring } \\
\square\end{array}$ & $\begin{array}{l}\text { Exciting } \\
\square\end{array}$ & $\begin{array}{l}\text { Stimulating } \\
\square\end{array}$ \\
\hline
\end{tabular}




\begin{tabular}{|c|c|c|c|c|c|}
\hline \multicolumn{6}{|c|}{ Table 8: Contd., } \\
\hline Structure of the websites & Rigid & Inflexible & Easy & $\begin{array}{l}\text { Extensible } \\
\square\end{array}$ & $\begin{array}{l}\text { Flexible } \\
\square\end{array}$ \\
\hline $\begin{array}{l}\text { Reading Characters on the } \\
\text { Page }\end{array}$ & Hard & $\begin{array}{l}\text { Difficult } \\
\square\end{array}$ & $\begin{array}{l}\text { Not so difficult } \\
\square\end{array}$ & Simple & Easy \\
\hline $\begin{array}{l}\text { Organization } \\
\text { Information }\end{array}$ & Hard & $\begin{array}{l}\text { Difficult } \\
\square\end{array}$ & $\begin{array}{l}\text { Not so difficult } \\
\square\end{array}$ & Simple & Easy \\
\hline Sequence of Page & Hard & $\begin{array}{l}\text { Difficult } \\
\square\end{array}$ & $\begin{array}{l}\text { Not so difficult } \\
\square\end{array}$ & Simple & Easy \\
\hline Terminology is intuitive & $\begin{array}{l}\text { Never } \\
\square\end{array}$ & $\begin{array}{l}\text { Sometimes } \\
\square\end{array}$ & Forever & $\begin{array}{ll}\text { At } & \text { all } \\
\text { times } & \\
\square & \end{array}$ & Always \\
\hline $\begin{array}{l}\text { Position of messages on } \\
\text { screen }\end{array}$ & $\begin{array}{l}\text { Inconsistent } \\
\square\end{array}$ & $\begin{array}{l}\text { Inherent } \\
\square\end{array}$ & $\begin{array}{l}\text { Uniform } \\
\square\end{array}$ & $\begin{array}{l}\text { Coherent } \\
\square\end{array}$ & $\begin{array}{l}\text { Consistent } \\
\square\end{array}$ \\
\hline $\begin{array}{l}\text { Sound associated with } \\
\text { Website }\end{array}$ & $\begin{array}{l}\text { Detracts } \\
\text { Value } \\
\square\end{array}$ & $\begin{array}{l}\text { Less Value } \\
\square\end{array}$ & Neutral & $\begin{array}{l}\text { Sums of } \\
\text { Value } \square\end{array}$ & Adds Value \\
\hline
\end{tabular}

Table 9: Most Common Things that are Attempted to do on this Website

\begin{tabular}{|l|l|l|l|l|l|}
\hline \multicolumn{1}{|c|}{ Factor } & No Opinion & Poor & Average & Good & Excellent \\
\hline To learn about the University & $\square$ & $\square$ & $\square$ & $\square$ & $\square$ \\
\hline To find contact information & $\square$ & $\square$ & $\square$ & $\square$ & $\square$ \\
\hline To find research publications or reports & $\square$ & $\square$ & $\square$ & $\square$ & $\square$ \\
\hline To find under I post graduate courses or & $\square$ & $\square$ & $\square$ & $\square$ & $\square$ \\
\hline To find about Ph.D programmes & $\square$ & $\square$ & $\square$ & $\square$ & $\square$ \\
\hline To find out about library & $\square$ & $\square$ & $\square$ & $\square$ & $\square$ \\
\hline
\end{tabular}

Table 10: Features of URL

\begin{tabular}{|l|l|l|l|l|l|}
\hline \multicolumn{1}{|c|}{ Factor } & Poor & Fair & Good & Very Good & Excellent \\
\hline Accessibility & $\square$ & $\square$ & $\square$ & $\square$ & $\square$ \\
\hline Accuracy & $\square$ & $\square$ & $\square$ & $\square$ & $\square$ \\
\hline Authority & $\square$ & $\square$ & $\square$ & $\square$ & $\square$ \\
\hline Consistency & $\square$ & $\square$ & $\square$ & $\square$ & $\square$ \\
\hline Ease of use & $\square$ & $\square$ & $\square$ & $\square$ & $\square$ \\
\hline Permanence & $\square$ & $\square$ & $\square$ & $\square$ & $\square$ \\
\hline Timeless & $\square$ & $\square$ & $\square$ & $\square$ & $\square$ \\
\hline Uniqueness & $\square$ & $\square$ & $\square$ & $\square$ & $\square$ \\
\hline Usefulness & $\square$ & $\square$ & $\square$ & $\square$ & $\square$ \\
\hline
\end{tabular}

Table 11: Website Design, Navigation and Content Organization

\begin{tabular}{|l|l|l|l|l|l|}
\hline \multicolumn{1}{|c|}{ Factor } & Poor & Fair & Good & $\begin{array}{c}\text { Very } \\
\text { Good }\end{array}$ & Excellent \\
\hline Individual pages are well designed & $\square$ & $\square$ & $\square$ & $\square$ & $\square$ \\
\hline This website is visually appealing & $\square$ & $\square$ & $\square$ & $\square$ & $\square$ \\
\hline Easy to move from one page to another & $\square$ & $\square$ & $\square$ & $\square$ & $\square$ \\
\hline $\begin{array}{l}\text { Navigation is very easy through the links or menu } \\
\text { which is easily recognizable. }\end{array}$ & $\square$ & $\square$ & $\square$ & $\square$ \\
\hline $\begin{array}{l}\text { The overall organization of the site is easy to } \\
\text { understand and hence easy to find desired information }\end{array}$ & $\square$ & $\square$ & $\square$ & $\square$ & $\square$ \\
\hline $\begin{array}{l}\text { The content of the website met my expectations and } \\
\text { interest }\end{array}$ & $\square$ & $\square$ & $\square$ & $\square$ & $\square$ \\
\hline $\begin{array}{l}\text { Language and terminology used in this website is clear } \\
\text { and familiar }\end{array}$ & $\square$ & $\square$ & $\square$ & $\square$ & $\square$ \\
\hline
\end{tabular}




\begin{tabular}{|l|l|l|l|l|l|}
\hline \multicolumn{3}{|c|}{ Table 11: Contd., } & & $\square$ \\
\hline $\begin{array}{l}\text { I was able to complete my task in a reasonable amount } \\
\text { of time }\end{array}$ & $\square$ & $\square$ & $\square$ & $\square$ \\
\hline $\begin{array}{l}\text { The website is allowing to open any new browser } \\
\text { windows when } \\
\text { I am moving around }\end{array} \square$ & $\square$ & $\square$ & $\square$ & $\square$ \\
\hline Likely to use this website in the future & $\square$ & $\square$ & $\square$ & $\square$ & $\square$ \\
\hline Maintained and up-to-date & $\square$ & $\square$ & $\square$ & $\square$ & $\square$ \\
\hline
\end{tabular}

Table 12: User Interface Design

\begin{tabular}{|l|l|l|l|l|l|}
\hline \multicolumn{1}{|c|}{ Factor } & $\begin{array}{c}\text { Strongly } \\
\text { disagree }\end{array}$ & $\begin{array}{c}\text { Dis } \\
\text { agree }\end{array}$ & $\begin{array}{c}\text { Un } \\
\text { decided }\end{array}$ & $\begin{array}{c}\text { Agree } \\
\begin{array}{c}\text { Strongly } \\
\text { Agree }\end{array}\end{array}$ \\
\hline The website's interface design is attractive & $\square$ & $\square$ & $\square$ & $\square$ & $\square$ \\
\hline The colours used in the website is comfortable & $\square$ & $\square$ & $\square$ & $\square$ & $\square$ \\
\hline No feature that irritates the user & $\square$ & $\square$ & $\square$ & $\square$ & $\square$ \\
\hline This website has a consistent feel and look & $\square$ & $\square$ & $\square$ & $\square$ & $\square$ \\
\hline $\begin{array}{l}\text { This website does not contain too many Web } \\
\text { advertisements }\end{array}$ & $\square$ & $\square$ & $\square$ & $\square$ & $\square$ \\
\hline $\begin{array}{l}\text { The design makes sense and it is easy to learn how } \\
\text { to use it }\end{array}$ & $\square$ & $\square$ & $\square$ & $\square$ & $\square$ \\
\hline
\end{tabular}

Table 13: Opinion on Academic Information

\begin{tabular}{|l|l|l|l|l|l|}
\hline \multicolumn{1}{|c|}{ Factor } & $\begin{array}{c}\text { Strongly } \\
\text { disagree }\end{array}$ & Disagree & undecided & Agree $\begin{array}{c}\text { Strongly } \\
\text { Agree }\end{array}$ \\
\hline It provides current information & $\square$ & $\square$ & $\square$ & $\square$ & $\square$ \\
\hline $\begin{array}{l}\text { It provides hyperlinks to related } \\
\text { information }\end{array}$ & $\square$ & $\square$ & $\square$ & $\square$ & $\square$ \\
\hline It is convenient to locate the sources & $\square$ & $\square$ & $\square$ & $\square$ & $\square$ \\
\hline It is easy to copy and edit & $\square$ & $\square$ & $\square$ & $\square$ & $\square$ \\
\hline It is freely available on the Web & $\square$ & $\square$ & $\square$ & $\square$ & $\square$ \\
\hline $\begin{array}{l}\text { It can be accessed any time / any } \\
\text { where }\end{array}$ & $\square$ & $\square$ & $\square$ & $\square$ & $\square$ \\
\hline
\end{tabular}

Table 14: General Information about Library Website

\begin{tabular}{|l|l|l|l|l|l|}
\hline \multicolumn{1}{|c|}{ Factor } & $\begin{array}{l}\text { Strongly } \\
\text { disagree }\end{array}$ & Disagree & Undecided & $\begin{array}{c}\text { Agree } \\
\text { Library is to have separate website / Web page }\end{array} \square$ & $\begin{array}{c}\text { Strongly } \\
\text { Agree }\end{array}$ \\
\hline Library website have separate site / sub-site & $\square$ & $\square$ & $\square$ & $\square$ \\
\hline $\begin{array}{l}\text { Library website have multiple web pages / single web } \\
\text { page }\end{array}$ & $\square$ & $\square$ & $\square$ & $\square$ & $\square$ \\
\hline $\begin{array}{l}\text { Hyperlink for library website / Web page (s) appear } \\
\text { under Facilities }\end{array}$ & $\square$ & $\square$ & $\square$ & $\square$ & $\square$ \\
\hline $\begin{array}{l}\text { Library website / Web page(s) provide updated } \\
\text { information }\end{array}$ & $\square$ & $\square$ & $\square$ & $\square$ & $\square$ \\
\hline
\end{tabular}

Table 15: Activities Organised Information in Website

\begin{tabular}{|l|l|l|l|l|l|}
\hline \multicolumn{1}{|c|}{ Factor } & Strongly disagree & Disagree & undecided & Agree & Strongly Agree \\
\hline Conferences / Training / Workshops & $\square$ & $\square$ & $\square$ & $\square$ & $\square$ \\
\hline Educational Courses & $\square$ & $\square$ & $\square$ & $\square$ & $\square$ \\
\hline Literacy Programmes & $\square$ & $\square$ & $\square$ & $\square$ & $\square$ \\
\hline User Orientation & $\square$ & $\square$ & $\square$ & $\square$ & $\square$ \\
\hline Refresher Course & $\square$ & $\square$ & $\square$ & $\square$ & $\square$ \\
\hline
\end{tabular}


Table 16: Evaluating the Efficiency

\begin{tabular}{|l|l|l|l|}
\hline \multicolumn{1}{|c|}{ Factor } & Dis agree & \multicolumn{1}{c|}{$\begin{array}{c}\text { No } \\
\text { Opinion }\end{array}$} & Agree \\
\hline It is easy to find the information & $\square$ & $\square$ & $\square$ \\
\hline It was easy to use the site on your first visit & $\square$ & $\square$ & $\square$ \\
\hline It is easy to navigate through this website & $\square$ & $\square$ & $\square$ \\
\hline The design of this website is appropriate & $\square$ & $\square$ & $\square$ \\
\hline Clicking on links takes me to what I expect & $\square$ & $\square$ & $\square$ \\
\hline The text is clear and easy to read & $\square$ & $\square$ & $\square$ \\
\hline The site takes a long time to download & $\square$ & $\square$ & $\square$ \\
\hline The site has been updated frequently & $\square$ & $\square$ & $\square$ \\
\hline $\begin{array}{l}\text { The organization of information on the website } \\
\text { pages is clear }\end{array}$ & $\square$ & $\square$ & $\square$ \\
\hline
\end{tabular}

Table 17: Opinion about URL

\begin{tabular}{|c|c|c|c|c|c|}
\hline Factor & $\begin{array}{l}\text { Strongly } \\
\text { disagree }\end{array}$ & Disagree & $\begin{array}{c}\text { Un } \\
\text { decided }\end{array}$ & Agree & $\begin{array}{l}\text { Strongly } \\
\text { Agree }\end{array}$ \\
\hline Reliable & $\square$ & $\square$ & $\square$ & $\square$ & $\square$ \\
\hline Authenticated & $\square$ & $\square$ & $\square$ & $\square$ & $\square$ \\
\hline Available in Future & $\square$ & $\square$ & $\square$ & $\square$ & $\square$ \\
\hline Standard Methods to Cite the URLs & $\square$ & $\square$ & $\square$ & $\square$ & $\square$ \\
\hline Easy to Locate the URLs & $\square$ & $\square$ & $\square$ & $\square$ & $\square$ \\
\hline $\begin{array}{l}\text { Commonly Available at the Same URL's } \\
\text { Address }\end{array}$ & $\square$ & $\square$ & $\square$ & $\square$ & $\square$ \\
\hline $\begin{array}{l}\text { Need Some Permanent Identification for } \\
\text { Evert Web Document }\end{array}$ & $\square$ & $\square$ & $\square$ & $\square$ & $\square$ \\
\hline Have Permanent Identification Address & $\square$ & $\square$ & $\square$ & $\square$ & $\square$ \\
\hline
\end{tabular}

Table 18: Reason for Inaccessibility

\begin{tabular}{|l|l|l|l|l|l|}
\hline \multicolumn{1}{|c|}{ Factor } & $\begin{array}{l}\text { Strongly } \\
\text { disagree }\end{array}$ & Disagree & $\begin{array}{c}\text { Un } \\
\text { decided }\end{array}$ & Agree & $\begin{array}{c}\text { Strongly } \\
\text { Agree }\end{array}$ \\
\hline Low bandwidth & $\square$ & $\square$ & $\square$ & $\square$ & $\square$ \\
\hline Misspelled in URLs & $\square$ & $\square$ & $\square$ & $\square$ & $\square$ \\
\hline Http errors & $\square$ & $\square$ & $\square$ & $\square$ & $\square$ \\
\hline Server down & $\square$ & $\square$ & $\square$ & $\square$ & $\square$ \\
\hline $\begin{array}{l}\text { URL is currently } \\
\text { inactive }\end{array}$ & $\square$ & $\square$ & $\square$ & $\square$ & $\square$ \\
\hline Lengthy URLs & $\square$ & $\square$ & $\square$ & $\square$ & $\square$ \\
\hline
\end{tabular}

Table 19: Performance Effectiveness

\begin{tabular}{|l|l|l|l|l|l|}
\hline \multicolumn{1}{|c|}{ Factor } & $\begin{array}{c}\text { Strongly } \\
\text { disagree }\end{array}$ & Disagree & $\begin{array}{c}\text { Un } \\
\text { decided }\end{array}$ & Agree & $\begin{array}{c}\text { Strongly } \\
\text { Agree }\end{array}$ \\
\hline $\begin{array}{l}\text { Less time to download a file or open a } \\
\text { page }\end{array}$ & $\square$ & $\square$ & $\square$ & $\square$ & $\square$ \\
\hline $\begin{array}{l}\text { Distinguish between visited and not } \\
\text { visited links }\end{array}$ & $\square$ & $\square$ & $\square$ & $\square$ & $\square$ \\
\hline access this website most of the time & $\square$ & $\square$ & $\square$ & $\square$ & $\square$ \\
\hline Must respond to the actions as expected & $\square$ & $\square$ & $\square$ & $\square$ & $\square$ \\
\hline Must be efficient to use & $\square$ & $\square$ & $\square$ & $\square$ & $\square$ \\
\hline Provides clear and useful messages & $\square$ & $\square$ & $\square$ & $\square$ & $\square$ \\
\hline
\end{tabular}


Table 20: Educational Purpose of Website

\begin{tabular}{|l|l|l|l|l|l|}
\hline \multicolumn{1}{|c|}{ Factor } & $\begin{array}{c}\text { Strongly } \\
\text { disagree }\end{array}$ & Disagree & $\begin{array}{c}\text { Un } \\
\text { decided }\end{array}$ & Agree & $\begin{array}{c}\text { Strongly } \\
\text { Agree }\end{array}$ \\
\hline $\begin{array}{l}\text { I can easily access the registration page and I can easily } \\
\text { register for semester. }\end{array}$ & $\square$ & $\square$ & $\square$ & $\square$ & $\square$ \\
\hline $\begin{array}{l}\text { When I need to register, the website provides } \\
\text { information about what the courses are offered and } \\
\text { who is teaching the courses. }\end{array}$ & $\square$ & $\square$ & $\square$ & $\square$ & $\square$ \\
\hline $\begin{array}{l}\text { This website is regularly updated in terms of personnel } \\
\text { and course information in order to keep their } \\
\text { information up-to-date. }\end{array}$ & $\square$ & $\square$ & $\square$ & $\square$ & $\square$ \\
\hline $\begin{array}{l}\text { I can easily contact with my instructors because this } \\
\text { website provides information about instructors office } \\
\text { location and hours and e-mail addresses. }\end{array}$ & $\square$ & $\square$ & $\square$ & $\square$ & $\square$ \\
\hline $\begin{array}{l}\text { This website suffers from problems during registration } \\
\text { process for students. }\end{array}$ & $\square$ & $\square$ & $\square$ & $\square$ & $\square$ \\
\hline $\begin{array}{l}\text { I know who I can contact for more information about } \\
\text { anything in this website. }\end{array}$ & $\square$ & $\square$ & $\square$ & $\square$ & $\square$ \\
\hline
\end{tabular}

Table 21: Content Organisation of Library

\begin{tabular}{|l|l|l|l|l|l|}
\hline \multicolumn{1}{|c|}{ Factor } & Poor & Fair & Good & $\begin{array}{c}\text { Very } \\
\text { Good }\end{array}$ & Excellent \\
\hline Introduction or historical details of the library & $\square$ & $\square$ & $\square$ & $\square$ & $\square$ \\
\hline Mission statement /aim/ objective/ purpose of the library & $\square$ & $\square$ & $\square$ & $\square$ & $\square$ \\
\hline Library collection information & $\square$ & $\square$ & $\square$ & $\square$ & $\square$ \\
\hline $\begin{array}{l}\text { electronic resources (e-journals \& databases, etc.) subscribed by } \\
\text { the library }\end{array}$ & $\square$ & $\square$ & $\square$ & $\square$ & $\square$ \\
\hline $\begin{array}{l}\text { Electronic resources (e-journals \& databases, etc.) subscribed by } \\
\text { the library accessed online }\end{array}$ & $\square$ & $\square$ & $\square$ & $\square$ & $\square$ \\
\hline $\begin{array}{l}\text { Information regarding print / current journals subscribed by the } \\
\text { library }\end{array}$ & $\square$ & $\square$ & $\square$ & $\square$ & $\square$ \\
\hline $\begin{array}{l}\text { information regarding e-books (ebrary) subscribed by the } \\
\text { library }\end{array}$ & $\square$ & $\square$ & $\square$ & $\square$ & $\square$ \\
\hline Ph.D. theses / dissertations to the users of the library & $\square$ & $\square$ & $\square$ & $\square$ & $\square$ \\
\hline Institutional repositories & $\square$ & $\square$ & $\square$ & $\square$ & $\square$ \\
\hline Web OPAC / Online catalogue & $\square$ & $\square$ & $\square$ & $\square$ & $\square$ \\
\hline
\end{tabular}
-:000:- 